\title{
Association between estimated blood glucose levels and glycated hemoglobin levels
}

\author{
Seon-Ah Cha and Seung-Hyun Ko
}

Division of Endocrinology and Metabolism, Department of Internal Medicine, College of Medicine, The Catholic University of Korea, Seoul, Korea

Received: April 13, 2016

Accepted: April 19, 2016

\section{Correspondence to}

\section{Seung-Hyun Ko, M.D.}

Division of Endocrinology and Metabolism, Department of Internal Medicine, College of Medicine, St. Vincent's Hospital, The Catholic University of Korea, 93 Jungbu-daero, Paldal-gu, Suwon 16247, Korea

Tel: +82-31-249-8155

Fax: +82-31-253-8898

E-mail:kosh@catholic.ac.kr

\section{See Article on Page 535-542}

For patients with diabetes, adequate glycemic control within a target range is key to preventing diabetes-related microvascular or macrovascular complications [1]. Many large epidemiological studies have highlighted the importance of intensive glucose control for patients with diabetes, especially for young subjects without diabetic complications who have been recently diagnosed [2]. For strict glycemic control, active lifestyle modification should be initiated as soon as possible after a diabetes diagnosis, and aggressive medical treatment using oral hypoglycemic agents and insulin injection should follow. To achieve glycemic target goals and enhance adherence to insulin therapy, a "patient-centered approach" is key [3]. This includes patient involvement in medical decision-making, a mutual exchange of information, and collaborative deliberation on options in order to reach a consensus on a patient's lifestyle choices and an appropriate therapeutic course of action [4]. To help patients make decisions about their own diabetic care, knowledge of their glycemic control status is essential. Therefore, given the options in diabetes education, glycemic measure methods (such as skills in self-monitoring of blood glucose [SMBG] and hemoglobin A1c $\left[\mathrm{AlC}_{1} \mathrm{C}\right]$ ) should be emphasized so that patients understand their glycemic control status and can help to prevent hypoglycemia [5].

Simple markers of glycemia, such as glycated proteins $\left(\mathrm{A}_{1} \mathrm{C}\right.$, glycated albumin, fructosamine) and 1,5-anhydroglucitol have broad clinical utility in the evaluation of a patient's glycemic control status (Table 1) [6-8]. There are two main techniques to assess the effectiveness of a management plan on glycemic control: SMBG and $\mathrm{AlC}$ measurement [9]. Solid knowledge of the glycemic control status of a patient with diabetes is very important for the initiation of hypoglycemic agents, dose adjustment of their medication, prevention of hypoglycemia, and guidance in treatment decisions [9]. In addition, the Korean Diabetes Association recommends regular $\mathrm{A} 1 \mathrm{C}$ testing every 3 to 6 months according to a patient's clinical situation [10]. The $\mathrm{AlC}_{1} \mathrm{C}$ is a simple, reproducible test that has an established association with risks for long-term diabetic complications in epidemiologic studies and clinical trials, and it has been used as a diagnostic criterion for diabetes after qualified standardization $[11,12]$. The $\mathrm{A} 1 \mathrm{C}$ results are expressed as a percentage of glycated hemoglobin, which does not hold much appeal for patients because it is not easily understood. Translating the 
Table 1. Measures of glycemia

\begin{tabular}{|c|c|c|c|c|}
\hline Method & Sampling site & Unit & $\begin{array}{l}\text { Monitoring } \\
\text { interval }\end{array}$ & Remark \\
\hline Continuous glucose monitoring & Interstitial fluid & $\mathrm{mg} / \mathrm{dL}$ & Every 5 min & $\begin{array}{l}\text { Need calibration } \\
\text { Use portable device }\end{array}$ \\
\hline Self-monitoring of blood glucose & Capillary blood & $\mathrm{mg} / \mathrm{dL}$ & Real time & Finger stick using glucometer \\
\hline Hemoglobin Aic & $\begin{array}{l}\text { Venous blood } \\
\text { (plasma, serum) }\end{array}$ & $\%$ & 3 mon & Need standardized assay \\
\hline $\begin{array}{l}\text { Glycated proteins } \\
\text { (fructosamine, glycated albumin) }\end{array}$ & Serum & $\mathrm{mmol} / \mathrm{L}, \%$ & $2-4 \mathrm{wk}$ & $\begin{array}{l}\text { Correlation with eAG is } \\
\text { not clear; not affected by anemia }\end{array}$ \\
\hline 1,5-Anhydroglucitol & Serum & $\mathrm{mg} / \mathrm{mL}$ & Several days-2 wk & $\begin{array}{l}\text { Cannot be used with SGLT2 } \\
\text { inhibitor use }\end{array}$ \\
\hline
\end{tabular}

eAG, estimated average glucose; SGLT2, sodium-glucose co-transporter 2.

$\mathrm{AlC}_{1} \mathrm{value}$ into an estimated average glucose (eAG) level is more practical and much easier for patients to understand. Therefore, it would be very practical if healthcare providers could predict mean glucose levels (i.e., eAG level) from a single blood sample test $\left(\mathrm{AlC}_{1} \mathrm{C}\right.$ rather than through troublesome, multiple finger-stick glucose monitoring. Regarding its clinical usefulness, running comparisons between SMBG (or frequently measured BG levels) and $\mathrm{A}_{1} \mathrm{C}$ values would be beneficial to determining the efficiency of this measure.

Some studies have tried to define the relationship between $\mathrm{A}_{1} \mathrm{C}$ levels and average glucose levels. In the A1C-Derived Average Glucose (ADAG) study, a total of 507 subjects, including type 1 diabetics, type 2 diabetics, and non-diabetics from 10 international centers were evaluated. The average glucose level was calculated from 2,700 glucose values based on continuous glucose monitoring with a 7-point daily SMBG [13]. The relationship between $\mathrm{AlC}$ and eAG is described by the formula eAG $=28.7 \times \mathrm{AlC}-46.7$. This means that every $1 \%$ increase in $\mathrm{A} 1 \mathrm{C}$ corresponds to an increase of approximately $29 \mathrm{mg} /$ dL in eAG. This formula was adopted by the American Diabetes Association and is available at http://professional.diabetes.org/eAG [9]. The formula was calculated based on quarterly $\mathrm{AlC}$ data and corresponding 7-point capillary BG profiles from the Diabetes Control and Complications Trial (DCCT). This trial included 1,441 subjects with type 1 diabetes, and the relationship between mean plasma glucose (MPG) and $\mathrm{AlC}_{1} \mathrm{C}$ was determined to be: $\mathrm{MPG}(\mathrm{mg} / \mathrm{dL})=35.6 \times \mathrm{AlC}-77.3$ [14] .

Regarding the present study [15], I would like to ex- press my appreciation for the authors' efforts. They provided valuable evidence concerning the association between mean BG levels derived from oral glucose tolerance tests and $\mathrm{AlC}_{1} \mathrm{C}$; this is the first for studies on the Korean population. The study included 1,000 subjects (391 males, 30 to 64 years old) with average serum glucose levels measured at $0,30,60$, and 120 minutes after loading with $75 \mathrm{~g}$ of glucose. They estimated that a $1 \%$ increase in the $\mathrm{AlClevel} \mathrm{was} \mathrm{associated} \mathrm{with} \mathrm{a} \mathrm{50-mg/dL}$ increase in the mean glucose level (mean glucose [mg/ $d L]=49.4 \times$ hemoglobin A1c [\%] - 149.6). In this study, the correlation coefficient was somewhat lower than in previous studies.

There are some notable points of discussion regarding the results and clinical implications of this impressive study. As the authors described in their Discussion section, there were only 64 patients with diabetes (identified as those with $\mathrm{AlC}$ levels higher than 6.5\%), which was too small a number to estimate the eAG for patients with diabetes. In this study, a single instance of 75-g glucose loading after 8 hours of fasting was used instead of a mixed meal stimulation before and after each meal; this is the process usually used to measure SMBG. Therefore, the glucose sampling time and site, as well as the serum glucose and capillary glucose concentrations, also differed from other studies. I suggest that this is one of the main reasons for the discrepancy between the DCCT cohort or ADAG studies and this Cardiovascular and Metabolic Diseases Etiology Research Center (CMERC) study cohort.

Frequent discordances between eAG and self-mon- 
itored mean BG levels have recently been reported. Chalew et al. [16] showed that eAG is often over- or underestimated by $28.7 \mathrm{mg} / \mathrm{dL}$ in approximately $33 \%$ of patients with type 1 diabetes. In addition, eAG often underestimates the mean BG in patients with type 1 diabetes with a low hemoglobin glycation index and overestimates mean BG in those with a high hemoglobin glycation index [17]. A study by the Juvenile Diabetes Research Foundation Continuous Glucose Monitoring Study Group included 252 subjects with type 1 diabetes and showed substantial individual variability between the measured and calculated mean BG concentrations [18]. In the Durability of Basal Versus Lispro Mix 75/25 Insulin Efficacy (DURABLE) trial, a study that included 1,879 participants with type 2 diabetes aged 30 to 80 years from 11 countries, eAG overestimated the actual mean BG at a mean SMBG level of $\leq 210 \mathrm{mg} / \mathrm{dL}$; at $>210 \mathrm{mg} / \mathrm{dL}$, eAG underestimated the actual BG levels [19]. In a study comparing the slopes of the linear correlations between $\mathrm{AlC}_{1}$ and CGM-measured mean glucose generated from the ADAG study data in an older population with diabetes, the two correlation coefficients were significantly different from each other [20]. The eAG is clinically practical and easily understood by patients with diabetes; however, it still shows some discrepancies across study populations, type of diabetes, glucose monitoring method, age range, and ethnicity. More clinical evidence must be accumulated and a consensus must be reached on these varying methods.

In conclusion, $\mathrm{AlC}$ is highly accurate and precise and has become standardized. Although eAG is easily understood, applicable, and practical in clinical settings, eAG does not yet seem able to replace ArC. Caution is also needed in the interpretation of $\mathrm{AlC}$ levels in patients with an unstable glycemic control status, pregnancy, steroid treatment, anemia, treatment with medications and vitamins, and renal impairment. To avoid clinically significant discordance between the calculated average glucose and a patient's own self-monitored mean BG level, additional studies are warranted.

\section{Conflict of interest}

No potential conflict of interest relevant to this article was reported.

\section{REFERENCES}

1. The Diabetes Control and Complications Trial Research Group. The effect of intensive treatment of diabetes on the development and progression of long-term complications in insulin-dependent diabetes mellitus. N Engl J Med 1993;329:977-986.

2. UK Prospective Diabetes Study (UKPDS) Group. Intensive blood-glucose control with sulphonylureas or insulin compared with conventional treatment and risk of complications in patients with type 2 diabetes (UKPDS 33). Lancet 1998;352:837-853.

3. Inzucchi SE, Bergenstal RM, Buse JB, et al. Management of hyperglycemia in type 2 diabetes: a patient-centered approach: position statement of the American Diabetes Association (ADA) and the European Association for the Study of Diabetes (EASD). Diabetes Care 2012;35:1364-1379.

4. Tsapas A, Matthews DR. N of 1 trials in diabetes: making individual therapeutic decisions. Diabetologia 2008;51:921-925.

5. Yong YM, Shin KM, Lee KM, et al. Intensive individualized reinforcement education is important for the prevention of hypoglycemia in patients with type 2 diabetes. Diabetes Metab J 2015;39:154-163.

6. Suh S, Kim JH. Glycemic variability: how do we measure it and why is it important? Diabetes Metab J 2015;39:273282.

7. Koga M. 1,5-Anhydroglucitol and glycated albumin in glycemia. Adv Clin Chem 2014;64:269-301.

8. Goldstein DE, Little RR, Lorenz RA, et al. Tests of glycemia in diabetes. Diabetes Care 2004;27:1761-1773.

9. American Diabetes Association. 5. Glycemic targets. Diabetes Care 2016;39 Suppl 1:S39-S46.

10. Ko SH, Kim SR, Kim DJ, et al. 2011 Clinical practice guidelines for type 2 diabetes in Korea. Diabetes Metab J 2011;35:431-436.

11. The Diabetes Control and Complications Trial Research Group. The relationship of glycemic exposure ( $\mathrm{HbA1c}$ ) to the risk of development and progression of retinopathy in the diabetes control and complications trial. Diabetes 1995;44:968-983.

12. Little RR, Rohlfing CL, Wiedmeyer HM, et al. The national glycohemoglobin standardization program: a five-year progress report. Clin Chem 2001;47:1985-1992.

13. Nathan DM, Kuenen J, Borg R, et al. Translating the $\mathrm{AlC}_{1}$ assay into estimated average glucose values. Diabetes 
Care 2008;31:1473-1478.

14. Rohlfing CL, Wiedmeyer HM, Little RR, England JD, Tennill A, Goldstein DE. Defining the relationship between plasma glucose and $\mathrm{HbA}(\mathrm{ic})$ : analysis of glucose profiles and $\mathrm{HbA}(\mathrm{1c})$ in the Diabetes Control and Complications Trial. Diabetes Care 2002;25:275-278.

15. Kim HJ, Kim YG, Park JS, Ahn YH, Ha KH, Kim DJ. Association between blood glucose level derived using the oral glucose tolerance test and glycated hemoglobin level. Korean J Intern Med 2016;31:535-542.

16. Chalew SA, Hempe JM, McCarter R. Clinically significant disagreement between mean blood glucose and estimated average glucose in two populations: implications for diabetes management. J Diabetes Sci Technol 2009;3:11281135 .

17. Hempe JM, Soros AA, Chalew SA. Estimated average glucose and self-monitored mean blood glucose are discordant estimates of glycemic control. Diabetes Care 2010;33:1449-1451.

18. Juvenile Diabetes Research Foundation Continuous Glucose Monitoring Study Group, Wilson DM, Xing D, et al. Hemoglobin Alc and mean glucose in patients with type 1 diabetes: analysis of data from the Juvenile Diabetes Research Foundation continuous glucose monitoring randomized trial. Diabetes Care 2011;34:540-544.

19. Wolffenbuttel BH, Herman WH, Gross JL, Dharmalingam $\mathrm{M}$, Jiang $\mathrm{HH}$, Hardin DS. Ethnic differences in glycemic markers in patients with type 2 diabetes. Diabetes Care 2013;36:2931-2936.

20. Munshi MN, Segal AR, Slyne C, Samur AA, Brooks KM, Horton ES. Shortfalls of the use of $\mathrm{HbArC}$-derived eAG in older adults with diabetes. Diabetes Res Clin Pract 2015;110:60-65. 